\title{
Folic acid supplementation and risk for congenital hydrocephalus in China
}

\author{
Jufen Liu ${ }^{1,2}$ (1), Zhiwen $\mathrm{Li}^{1,2, *, \dagger}$, Rongwei Ye ${ }^{1,2, \dagger}$, Aiguo Ren ${ }^{1,2}$ and Jianmeng Liu ${ }^{1,2}$ (1) \\ 'Institute of Reproductive and Child Health, Key Laboratory of Reproductive Health, National Health Commission of the \\ People's Republic of China, Peking University, 38 College Rd, Haidian District, Beiijing 100191, People's Republic of \\ China: ${ }^{2}$ Department of Epidemiology and Biostatistics, School of Public Health, Peking University, Beijing, People's \\ Republic of China
}

Submitted 12 August 2020: Final revision received 22 December 2020: Accepted 18 January 2021: First published online 26 January 2021

\begin{abstract}
Objective: We examined whether folic acid (FA) supplementation prevented congenital hydrocephalus $(\mathrm{CH})$ in more than 200000 births in China.

Design: A large population-based cohort study.

Setting: All births at 20 complete gestational weeks, including live births, stillbirths and pregnancy terminations, and all structural birth defects regardless of gestational week were recorded. The prevalence of births with $\mathrm{CH}$ was classified by maternal characteristics and FA supplementation. $\mathrm{CH}$ was diagnosed in accordance with code 742.3 of the International Classification of Diseases, Ninth Revision, Clinical Modification, while non-neural tube defect (NTD) CH refers to $\mathrm{CH}$ without anencephaly (740), spina bifida (741) or encephalocele (742.0). Participants: A total of 247831 pregnant women who delivered with known outcomes were included.

Results: A total of 206 cases of $\mathrm{CH}$ ( 0.83 per 1000 births) and 170 cases of non-NTD $\mathrm{CH}$ (0.69 per 1000 births) were recorded in the study. The prevalence of $\mathrm{CH}$ and non-NTD CH was higher in women in the no supplementation group than those in the FA supplementation group $(0.92$ and $0.72 v .0 .75$ and 0.65 per 1000 births, respectively). FA supplementation during the periconceptional period significantly prevented $\mathrm{CH}(\mathrm{OR}=0 \cdot 29,95 \% \mathrm{CI} 0 \cdot 12,0 \cdot 69)$ and non-NTD CH $(\mathrm{OR}=0 \cdot 34,95 \% \mathrm{CI}$ $0 \cdot 12,0.97)$ in northern China, especially in a high-compliance group ( $\geq 80 \%$ ). Conclusions: Periconceptional FA supplementation did not significantly prevent $\mathrm{CH}$ overall in the current study. However, in the north of China with common maternal folate insufficiency, there was some evidence.
\end{abstract}

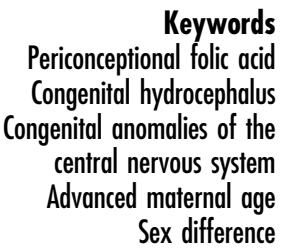

Malformations of the central nervous system (CNS), some of the most prevalent congenital anomalies, represent an important source of morbidity and mortality in children ${ }^{(1)}$. In lowincome countries, an estimated $29 \%$ of neonatal deaths related to visible congenital abnormalities are attributable to neural tube defects (NTD), which are also associated with lifelong disability $^{(2)}$. Besides NTD, congenital hydrocephalus $(\mathrm{CH})$ is another common major birth defect of the CNS, and $60 \%$ of $\mathrm{CH}$ is complicated with $\mathrm{NTD}^{(3)}$. $\mathrm{CH}$, which is characterised by an imbalance in cerebrospinal fluid secretion/absorption that results in the accumulation of fluid in the brain with consequential pathophysiology ${ }^{(4,5)}$, is an important cause of neurological morbidity and mortality in children ${ }^{(6)}$. The global

These authors contributed equally to this work prevalence of $\mathrm{CH}$ has remained stable over the past decade at 0.81 per 1000 births $^{(7)}$.

Periconceptional supplementation with folic acid (FA) among women of childbearing age can prevent NTD ${ }^{(8)}$, although the preventive effects on $\mathrm{CH}$ have been less studied. Animal studies have revealed that a deficiency in 10-formyl-tetrahydrofolate-dehydrogenase, a folate binding protein, in the nucleus of the brain and liver is linked to decreased DNA methylation, which could be a key factor in the developmental deficits associated with congenital and neonatal hydrocephalus ${ }^{(4,9)}$. Although the causes and mechanisms underlying the development of $\mathrm{CH}$ are not the same as those involved in NTD, folate and vitamin $\mathrm{B}_{12}$ have fundamental roles in the functioning of the CNS and in the prevention of disorders that affect its development ${ }^{(10)}$. 
Most $\mathrm{CH}$ is accompanied by NTD, and thus $\mathrm{CH}$ with non-NTD has been less studied. There are few reports of $\mathrm{CH}$ malformation according to maternal characteristics. Using a large-scale population-based surveillance study, we explored the epidemiology of $\mathrm{CH}$ and analysed preventive effects of FA supplementation on $\mathrm{CH}$ in China.

\section{Methods}

Methods of the original study have been described previously $^{(8,11)}$. Briefly, the Chinese Ministry of Health conducted a public health campaign to prevent NTD in twenty-one counties in southern China (located in Zhejiang and Jiangsu province) and northern China (located in Hebei province) in the early 1990s. A well-organised pregnancy monitoring system (PMS) was established to collect principal records on prenatal care and delivery. Data on the demographic variables and most of the covariates came from this system, with local maternal clinicians collecting the information. All women in the project counties who were preparing for marriage or who became pregnant were registered. The enrolled women were advised to take a pill containing 400 $\mu \mathrm{g}$ FA every day, starting at the time they registered with the PMS and continuing until the completion of their first trimester of pregnancy. Details on FA supplementation were recorded by local health care workers ${ }^{(8,11)}$. Detailed forms were used to record the number of tablets women took and the dates of all menstrual periods. Mothers' clinicians distributed bottles of the tablets, supervised the participants, retrieved the bottles and counted the remaining tablets every month. All births at 20 complete gestational weeks, including live births, stillbirths and pregnancy terminations, and all structural congenital anomalies regardless of gestational week were recorded ${ }^{(12)}$. To be included in the study, women had to be residents of the county, be registered with the PMS between October 1993 and September 1995 and deliver with a known outcome by 31 December 31 1996. Women who did not live in the county, did not give birth in the county or did not provide oral consent were excluded.

The project was approved by the institutional review boards of the US Centers for Disease Control and Prevention and Peking University Health Science Center. All women who took pills provided oral informed consent.

\section{Folic acid use: definition and compliance}

The classification and patterns of FA consumption were the same as previously reported ${ }^{(8)}$. Women who took FA pills at any time from the registration period until the end of the first trimester of pregnancy were classified as FA users. Women who did not agree to take FA or who were registered during the second trimester of pregnancy (i.e., did not have the opportunity to start taking FA by the end of the first trimester) were considered non-users. Three patterns of FA exposure were determined according to when women started and stopped taking FA: (1) periconceptional: initiation before the last menstrual period (LMP) and termination within the first trimester, (2) preconceptional: initiation and termination before the LMP and (3) postconceptional: initiation after the LMP and termination within the first trimester. Compliance was calculated as the number of pills actually consumed divided by the number of pills assigned. A subgroup of women who took more than $80 \%$ of their assigned pills was defined as the high-compliance group ${ }^{(8)}$.

\section{Identification of congenital hydrocephalus}

Congenital anomalies of the CNS were identified through a birth defects surveillance system ${ }^{(8,12)}$. Live-born infants with birth defects were included in the surveillance system if they had a gestational age of at least 20 weeks. Information on all pregnancies, even those $<20$ weeks' gestation that were electively terminated after a prenatal diagnosis of any birth defect, was also collected. Details of diagnoses were described previously; briefly, diagnoses of suspected defects were made based on photographs of affected fetuses and infants taken at birth or termination of pregnancy and on reviews of reports by several clinicians ${ }^{(11)}$. Diagnoses of birth defects were made according to the International Classification of Diseases, Ninth Revision, Clinical Modification. $\mathrm{CH}$ was diagnosed in accordance with code 742.3, while non-NTD CH refers to $\mathrm{CH}$ without anencephaly (740), spina bifida (741) or encephalocele $(742 \cdot 0)^{(3)}$.

\section{Statistical analysis}

The main effect of FA supplementation (exposure) on $\mathrm{CH}$ was estimated. Because our previous study revealed that only $40 \%$ of $\mathrm{CH}$ was non-NTD $\mathrm{CH}^{(3)}$, to comprehensively reflect $\mathrm{CH}$ status we included both $\mathrm{CH}$ and non-NTD $\mathrm{CH}$ in our study. As there were no reports in the literature on relative risk, we used the preventive effect of FA on NTD (i.e., the OR of the exposure group relative to the control group), $0 \cdot 8^{(8)}$, to estimate the incidence of $\mathrm{CH}$ in the control group at 0.81 per 1000 births $^{(7)}$. Assuming $\alpha=0.05$ (bilateral) and $\beta=0 \cdot 10$, according to case: control $=1: 2$, significant difference between two groups was $0.2 \%$, the sample sizes of the exposure group would be 68766 and sample size in control group was 137532 . A total of 247831 participants in our study could meet the statistical requirements. The sample size was calculated with PASS (version 20.0.3; NCSS).

We compared means using $t$ tests and distributions using $\chi^{2}$ tests. We calculated the rate of $\mathrm{CH}$ (i.e., the number of cases per 1000 pregnancies of at least 20 weeks' gestation) according to patterns of FA intake. We estimated risk ratios by dividing the risk for $\mathrm{CH}$ among the fetuses or infants of all women who took FA by the risk among the fetuses or infants of women who did not take FA. We also estimated risk ratios by dividing the risk among the fetuses or infants of women with periconceptional/preconceptional/postconceptional FA 
by the risk among the fetuses or infants of women who did not take FA. In logistic regression, the dependent variable was $\mathrm{CH}$ and the independent variable was FA supplementation; the main potentially confounding variables adjusted for in logistic regression included maternal age at delivery, ethnicity, education, occupation, parity and region. We ran the logistic regression separately for $\mathrm{CH}$ and non-NTD $\mathrm{CH}$. Missing data for continuous variables (age and $\mathrm{BMI}$ ) were replaced by the mean of the corresponding variable. Missing data for categorical variables (parity, ethnicity, education and occupation) were replaced by the most common value. Model assumptions and goodness of fit were evaluated with the omnibus test ( -2 log likelihood) and Hosmer and Lemeshow test. All data were analysed with SPSS (version 24.0; IBM).

\section{Results}

Among the 247831 participants, the mean age was 25.4 (SD 3.2) years; $85 \%$ of participants were younger than 30 years old. A total of $12.9 \%$ of subjects were from the north and $87.1 \%$ were from the south. Among both $\mathrm{CH}$ and non-NTD CH groups, $62 \%$ of mothers were farmers, $55-56 \%$ of mothers had finished junior high school and there were more male than female infants (Table 1). A total of $52.5 \%$ of women took FA during the periconceptional period. The proportion of FA supplementation was higher in the north $(58.2 \%)$ than the south $(51.7 \%)$. Women who took FA supplements were more likely to be primiparous and to have higher education. A full description of the subjects is available elsewhere ${ }^{(11)}$.

A total of 206 cases of $\mathrm{CH}$ and 170 cases of non-NTD CH were recorded in the study. The prevalence of $\mathrm{CH}$ was significantly higher in the north (1.31 per 1000 births) and among women older than 35 years ( 2.33 per 1000 births) than in the south and among younger women $(0.76$ and 0.82 per 1000 births, respectively). Differences in the prevalence of non-NTD $\mathrm{CH}$ among these groups did not reach statistical significance (Table 2).

The prevalence of $\mathrm{CH}$ was lower among women who took FA tablets than those who did not, although the difference was not significant. The prevalence of both $\mathrm{CH}(0.54$ per 1000 births) and non-NTD CH (0.38 per 1000 births) was significantly lower in the periconceptional FA supplementation group than among non-users (1.72 and 1.12 per 1000 births for $\mathrm{CH}$ and non-NTD $\mathrm{CH}$, respectively; Table 3). No significant difference for this effect was observed in the south. The data indicated that women in the high-compliance ( $\geq 80 \%$ ) FA supplementation group had a lower prevalence of $\mathrm{CH}$ compared with women in the low-compliance ( $<80 \%)$ group and non-users, although the difference was not statistically significant. Our study size had $99.9 \%$ power $(\alpha=0.05)$ to detect a decrease of $10 \%$ over the unexposed rate of 0.81 per 1000 for $\mathrm{CH}$.
Table 1 Description of $\mathrm{CH}$ by maternal characteristics in China, 1993-1996

\begin{tabular}{|c|c|c|c|c|c|c|}
\hline \multirow[b]{2}{*}{ Variable } & \multirow[b]{2}{*}{$n^{*}$} & \multirow[b]{2}{*}{$\%$} & \multicolumn{2}{|c|}{$\mathrm{CH}$} & \multicolumn{2}{|c|}{$\begin{array}{c}\text { Non-NTD } \\
\mathrm{CH}\end{array}$} \\
\hline & & & $n$ & $\%$ & $n$ & $\%$ \\
\hline \multicolumn{7}{|l|}{ Maternal age } \\
\hline$<35$ years & 245688 & $99 \cdot 1$ & 201 & $97 \cdot 6$ & 167 & 98.2 \\
\hline$\geq 35$ years & 2143 & 0.9 & 5 & $2 \cdot 4$ & 3 & 1.8 \\
\hline \multicolumn{7}{|l|}{ Occupation } \\
\hline Farmer & 152719 & 61.6 & 129 & $62 \cdot 6$ & 109 & 61.9 \\
\hline Factory worker & 76960 & $31 \cdot 1$ & 50 & $24 \cdot 3$ & 42 & 23.9 \\
\hline Other & 18152 & $7 \cdot 3$ & 27 & $13 \cdot 1$ & 25 & 14.2 \\
\hline \multicolumn{7}{|l|}{$\mathrm{BMI}\left(\mathrm{kg} / \mathrm{m}^{2}\right)$} \\
\hline$<18.5$ & 32061 & 12.9 & 19 & 9.2 & 16 & 9.4 \\
\hline $18 \cdot 5-23.9$ & 203168 & $82 \cdot 0$ & 177 & 85.9 & 144 & 84.7 \\
\hline $24-27 \cdot 9$ & 11569 & 4.7 & 10 & 4.9 & 10 & 5.9 \\
\hline$\geq 28$ & 1033 & 0.4 & 0 & 0 & 0 & 0 \\
\hline \multicolumn{7}{|l|}{ Education } \\
\hline $\begin{array}{l}\text { Senior high school } \\
\text { above }\end{array}$ & 25485 & $10 \cdot 3$ & 15 & $8 \cdot 1$ & 14 & $9 \cdot 2$ \\
\hline Junior high school & 148589 & $60 \cdot 0$ & 105 & $56 \cdot 5$ & 84 & $55 \cdot 3$ \\
\hline Elementary school & 66999 & $27 \cdot 0$ & 66 & 35.5 & 54 & 35.5 \\
\hline Missing & 6758 & 2.7 & & & & \\
\hline \multicolumn{7}{|l|}{ Ethnicity } \\
\hline Han & 239914 & 99.4 & 186 & 100 & 152 & 100 \\
\hline Others & 1561 & 0.6 & 0 & & 0 & \\
\hline Missing & 6356 & $2 \cdot 6$ & & & & \\
\hline \multicolumn{7}{|l|}{ Parity } \\
\hline Primiparous & 204987 & 82.7 & 150 & $82 \cdot 0$ & 125 & $80 \cdot 6$ \\
\hline Multiparity & 40214 & $16 \cdot 2$ & 33 & $18 \cdot 0$ & 30 & $19 \cdot 4$ \\
\hline Missing & 2630 & 1.1 & & & & \\
\hline \multicolumn{7}{|l|}{ Sex of infant } \\
\hline Male & 128566 & 51.9 & 105 & $51 \cdot 7$ & 90 & 53.9 \\
\hline Female & 118625 & 47.9 & 98 & 48.3 & 77 & $46 \cdot 1$ \\
\hline Missing & 640 & 0.2 & & & & \\
\hline \multicolumn{7}{|l|}{ Region } \\
\hline North & 31960 & 12.9 & 42 & $20 \cdot 4$ & 29 & $17 \cdot 1$ \\
\hline South & 215871 & $87 \cdot 1$ & 164 & $79 \cdot 6$ & 141 & 82.9 \\
\hline
\end{tabular}

$\mathrm{CH}$, congenital hydrocephalus.

${ }^{*}$ Due to missing data for variables, sum of values was not equal the total numbers.

Results for model assumptions revealed that the model had good fit, with both the -2 log likelihood and Hosmer and Lemeshow tests showing $P>0.05$ (online supplementary material, Supplemental Table 1). No overall preventive effect of FA on CH was observed in China. When we analysed further by region, we found that FA supplementation during the periconceptional period significantly prevented $\mathrm{CH}(\mathrm{OR}=0.29,95 \% \mathrm{CI} 0 \cdot 12,0.69)$ and non-NTD $\mathrm{CH}$ $(\mathrm{OR}=0.34,95 \% \mathrm{CI} 0 \cdot 12,0.97)$ in the north. Further analyses showed that higher compliance with FA supplementation $(\geq 80 \%$ ) significantly reduced the risk for $\mathrm{CH}$ $(\mathrm{OR}=0.25,95 \%$ CI $0.09,0.66)$ and non-NTD $\mathrm{CH}$ $(\mathrm{OR}=0.33,95 \% \mathrm{CI} 0 \cdot 10,1.03)$ in the north (Table 4).

\section{Discussion}

Although there is a consensus that periconceptional FA supplementation can reduce NTD, few studies have explored its effects on $\mathrm{CH}$, one of the most common congenital CNS abnormalities besides NTD. Our study 
Table 2 Prevalence of $\mathrm{CH}$ in China, 1993-1996, $n$ (per 1000 births)*

\begin{tabular}{|c|c|c|c|c|c|c|c|}
\hline \multirow[b]{2}{*}{ Variables } & \multirow[b]{2}{*}{$n^{*}$} & \multicolumn{2}{|c|}{$\mathrm{CH}$} & \multirow[b]{2}{*}{$P$} & \multicolumn{2}{|c|}{ Non-NTD CH } & \multirow[b]{2}{*}{$P$} \\
\hline & & $n$ & $\%$ & & $n$ & $\%$ & \\
\hline \multicolumn{8}{|l|}{ Region } \\
\hline North & 31960 & 42 & $1 \cdot 31$ & \multirow[t]{2}{*}{$0.001 \dagger$} & 29 & 0.91 & \multirow[t]{2}{*}{0.65} \\
\hline South & 215871 & 164 & 0.76 & & 141 & 0.65 & \\
\hline \multicolumn{8}{|l|}{ Sex of infant } \\
\hline Male & 128566 & 105 & 0.82 & \multirow[t]{2}{*}{0.935} & 90 & 0.70 & \multirow[t]{2}{*}{0.626} \\
\hline Female & 118625 & 98 & 0.83 & & 77 & 0.65 & \\
\hline \multicolumn{8}{|l|}{ Maternal age } \\
\hline$<35$ years & 245688 & 201 & 0.82 & \multirow[t]{3}{*}{$0.015 \dagger$} & 167 & 0.68 & \multirow[t]{3}{*}{0.205} \\
\hline$\geq 35$ years & 2143 & 5 & $2 \cdot 33$ & & 3 & 1.40 & \\
\hline Total & 247831 & 206 & 0.83 & & 170 & 0.69 & \\
\hline
\end{tabular}

$\mathrm{CH}$, congenital hydrocephalus.

*Due to missing data for variables, sum of values was not equal the total numbers. $\dagger P<0.05$, comparison between regions or maternal age groups.

provides new evidence that periconceptional FA supplementation can prevent $\mathrm{CH}$ in northern China, where there is a high prevalence of NTD.

Using data from a large population-based surveillance system and rigorous diagnosis of birth defects, we reported the epidemiology of $\mathrm{CH}$ and non-NTD $\mathrm{CH}$. $\mathrm{CH}$ was the most common CNS abnormality after NTD. In our study, NTD accounted for $58 \%$ (275/474) of all CNS abnormalities, followed by $\mathrm{CH}(36 \%, 170 / 474)$. Prevalence rates in the current study were 0.83 and 0.69 per 1000 live births for $\mathrm{CH}$ and non-NTD $\mathrm{CH}$, which is close to the global average $(0.81 \text { per } 1000 \text { live births })^{(7)}$, but higher than in European countries ( 0.47 per 1000 live births $)^{(13)}$ and the USA/Canada (0.68 per 1000 live births $)^{(6)}$ while it is lower than Africa (1.45 per 1000 live births) and Latin America (3.16 per 1000 live births) ${ }^{(14)}$. These prevalence rates are also higher than reports of around 0.7 per 1000 births in China for 1996-2004 ${ }^{(15)}, 0.62$ per 1000 births in 2005 and 0.42 per 1000 births for isolated $\mathrm{CH}$ in $2012^{(16)}$. The latter data are from a hospital-based birth defect surveillance system that only included births of 28 weeks' gestation or greater, whereas our study included all births at 20 complete gestational weeks and all structural abnormalities regardless of gestational week. Population-based surveillance systems that include large numbers of pregnant women can provide more complete data on outcomes ${ }^{(8)}$ and give more accurate estimations of the epidemiology of $\mathrm{CH}$. As the primary aim of this large cohort study was to identify NTD, abnormality of CNS was specially checked and recorded, non-NTD $\mathrm{CH}$ was recorded accordingly. The preventive effect of FA on NTD is well known, whereas the effect of $\mathrm{CH}$ is less frequently reported. Thus, our study adds important evidence on this topic, especially nonNTD CH was analysed separately and explored.

The prevalence of $\mathrm{CH}$ was significantly higher in the north than in the south: 1.31 compared with 0.76 per 1000 births, respectively. This is in line with the welldocumented difference in rates of NTD between the two regions, which could be due in part to differences in dietary FA intake ${ }^{(8)}$. As the original study did not collect information on diet, and it is impossible to examine individual blood sample in a large-scale population study, folate concentrations were examined in a cross-sectional survey in 2003 of women in their first trimester of pregnancy from one county and one city in both the north and south. Results showed that women in the north had less than half the folate concentration of women in the south (440.0 $\mathrm{v}$. $910 \cdot 4 \mathrm{mmol} / 1$, respectively) $)^{(17)}$.

Periconceptional FA supplementation can prevent $\mathrm{CH}$ in northern China. Starting to supplement before the LMP and continuing until the full first trimester was key; early discontinuation and late onset did not have preventive effects on $\mathrm{CH}$. As most $\mathrm{CH}$ was followed by NTD, periconceptional FA supplementation would prevent NTD and the subsequent $\mathrm{CH}$. Our study provides new evidence that non-NTD $\mathrm{CH}$ is also preventable with periconceptional FA supplementation. As FA supplementation has a strong impact on plasma folate concentrations, massive FA supplementation in northern China has significantly improved folate concentrations and reduced $\mathrm{CH}$ over time ${ }^{(3)}$. The cause as well as the heterogeneity of its mechanisms needs to be explored further. Earlier supplementation (before the LMP), increased supplementation frequency and more total days of supplementation are associated with increasing folate concentrations ${ }^{(18)}$. The current nationwide programme should ensure that supplementation begins before pregnancy and continues for a long enough time.

The causes of and mechanisms underlying the development of $\mathrm{CH}$ are not well understood. Genetic mutation ${ }^{(19)}$, folate imbalance ${ }^{(20)}$ and abnormal folate metabolism ${ }^{(9)}$ have all been reported as risk factors for $\mathrm{CH}$. An animal study revealed that FA deficiency induces antenatal hydrocephalus in rat ${ }^{(21)}$. In previous studies, FA supplementation was estimated through administrative data while lack of individual FA supplementation data in one study ${ }^{(6)}$ and another study revealed that folate fortification status was not associated with the incidence of hydrocephalus ${ }^{(7)}$. Our previous study, which relied on indirect evidence of birth defects and massive FA policy, revealed that FA supplementation was correlated with decreasing $\mathrm{CH}^{(3)}$. Using 
strict surveillance data on FA supplementation from a large population, the study provided direct, first-hand evidence of the preventive effect of FA on $\mathrm{CH}$ as well as non-NTD $\mathrm{CH}$ among women with folate insufficiency.

The present study has several strengths. First, it was based on a well-organised, population-based PMS that used quality control measures to ensure the collection of high-quality data on subjects and the diagnosis of birth defects. Birth defects were recorded regardless of gestational week, a more accurate process than hospital-based surveillance (which only covers $\geq 28$ weeks). A previous study revealed that pre-perinatal prevalence $(<28$ gestational weeks) was higher than perinatal prevalence $(>28$ gestational weeks) for both non-NTD and total $\mathrm{CH}^{(3)}$, which reflects an underreporting in hospital-based surveillance. Second, in our study, reliable data on FA supplementation were documented prospectively before the outcome of the pregnancy was known ${ }^{(8)}$. All FA supplements were supplied by the community intervention project; there were no over-the-counter FA supplements in China at that time. Detailed information on FA supplementation was recorded prospectively. If women consented to take FA, the pills were distributed at the time of registration. At the end of each month, health care workers recorded the dates of all menstrual periods and how many pills remained in each bottle. Supplementation started at the time of registration with the PMS and stopped at the end of the first trimester. FA supplementation was ultimately confirmed by local health care workers, who checked the number of pills taken at monthly home visits. Third, FA supplementation $(400 \mu \mathrm{g} / \mathrm{d})$ was the only intervention in the study. There was no market supply of FA supplements or multivitamins that included FA in the early 1990s in the study area, and there was no massive FA supplementation or food fortification among women of childbearing age in China ${ }^{(22)}$. Our study thus provides evidence of the effect of FA only on $\mathrm{CH}$ among the largest population studied to date.

Limitations of the study should also be noted. First, we did not collect information on the dietary folate intake of the women, which could have influenced the results. However, the study population had low folate levels, and there was no other FA fortification besides the current intervention. Second, we did not explore the effects of other potential confounding factors associated with FA use. For example, the women were not randomly assigned to the FA supplementation or no supplementation groups ${ }^{(8)}$, and systematic differences between the two groups may have influenced FA use. Women who did not take FA were slightly older than those who did and were more likely to have been pregnant before, while further stratified analysis according to age and the number of previous pregnancies did not influence the result. However, multivariate analyses adjusted by age and parity did not reveal different results. Other lifestyle factors such as smoking or alcohol consumption, husband characteristics may be considered in future study. Third, the supplementation period in the current 
Table $4 \mathrm{RR}$ and $95 \% \mathrm{Cl}$ for $\mathrm{CH}$ by folic acid supplementation and compliance in China, 1993-1996

\begin{tabular}{|c|c|c|c|c|c|c|c|c|}
\hline \multirow[b]{2}{*}{ Variable } & \multicolumn{4}{|c|}{ Crude RR } & \multicolumn{4}{|c|}{ Adjusted RR } \\
\hline & $\mathrm{CH}$ & $95 \% \mathrm{Cl}$ & Non-NTD CH & $95 \% \mathrm{Cl}$ & $\mathrm{CH}$ & $95 \% \mathrm{Cl}$ & Non-NTD CH & $95 \% \mathrm{Cl}$ \\
\hline \multicolumn{9}{|c|}{$\begin{array}{l}\text { Folic acid supplementation } \\
\text { (yes/no) }\end{array}$} \\
\hline North* & 0.59 & $0.32,1.09$ & 0.67 & $0.32,1.39$ & 0.55 & $0.29,1.05$ & 0.68 & $0.31,1.48$ \\
\hline South* & 0.87 & $0.64,1.18$ & 0.95 & $0.68,1.32$ & 0.88 & $0.64,1.22$ & 0.98 & $0.69,1.39$ \\
\hline Total† & 0.82 & $0.62,1.08$ & 0.90 & $0.67,1.22$ & 0.81 & $0.61,1.07$ & 0.92 & $0.67,1.26$ \\
\hline \multicolumn{9}{|c|}{ Periconceptional supplementation } \\
\hline North* & 0.31 & $0.13,0.73$ & 0.34 & $0.12,0.94$ & 0.29 & $0.12,0.69 \ddagger$ & 0.34 & $0.12,0.97 \ddagger$ \\
\hline$\geq 80 \%$ & 0.27 & $0.10,0.72$ & 0.33 & $0.11,1.00$ & 0.25 & $0.09, \cdot 0 \cdot 66 \ddagger$ & 0.33 & $0.10,1.03 \ddagger$ \\
\hline$<80 \%$ & 0.49 & $0.12,2 \cdot 12$ & 0.38 & $0.05,2.89$ & 0.48 & $0.11,2.09$ & 0.39 & $0.05,3.09$ \\
\hline South* & 0.94 & $0.66,1.39$ & $1 \cdot 12$ & $0.77,163$ & 0.96 & $0.66,1.39$ & $1 \cdot 16$ & $0.79,1.72$ \\
\hline Totalt & 0.79 & $0.57,1.10$ & 0.95 & $0.67,1.35$ & 0.76 & $0.54,1.07$ & 0.95 & $0.66,1.38$ \\
\hline
\end{tabular}

$\mathrm{RR}$, risk ratio; $\mathrm{CH}$, congenital hydrocephalus.

*Adjusted for age at pregnancy, BMI, parity, ethnicity, education, occupation.

†Adjusted for age at pregnancy, BMI, parity, ethnicity, education, occupation, and region.

†Refers to model 1-4, with good fit (see Model assumption details in online supplementary material, Supplemental Table 1).

study was from the time of registration in the PMS to the end of the first trimester of pregnancy, so we could only observe the potential effect of FA supplementation in the first trimester. Whether continued supplementation in the second or third trimesters would have preventive effects on $\mathrm{CH}$ requires further study.

Despite these limitations, our large sample size and use of population-based data as well as the individual data on FA supplementation reveal a correlation between periconceptional FA supplementation (until the first trimester) and $\mathrm{CH}$. In conclusion, periconceptional FA supplementation does not significantly prevent $\mathrm{CH}$ overall. However, in northern China of common maternal folate insufficiency, there is some evidence. Further study of the causes and underlying mechanisms of $\mathrm{CH}$ is needed to develop preventive measures.

\section{Acknowledgements}

Acknowledgements: The authors thank the staff members and participating women of the original trial. The authors also thank the anonymous reviewers for helpful comments. Financial support: This work was supported in part by the National Key Research and Development Program, Ministry of Science and Technology of the People's Republic of China (grant No. 2016YFC1000501) and Natural Science Foundation of China (No. 81373014 and No. 81202265). The original project was supported by a cooperative agreement between the US Centers for Disease Control and Prevention and Peking University (grant no. U01 DD000293). Conflict of interest: None. Authorship: J.F.L. conceptualised the study, analysed the data, drafted the initial manuscript, and reviewed and revised the manuscript. Z.L. and R.Y. conceptualised and designed the study, and critically revised the manuscript; A.R., R.Y. and J.M.L. coordinated and supervised data collection, reviewed and revised the manuscript. All authors read, reviewed and approved the final manuscript. All authors approved the final manuscript as submitted and agree to be accountable for all aspects of the work. Ethics of human subject participation: The current study was conducted according to the guidelines laid down in the Declaration of Helsinki and all procedures involving research study participants were approved by the institutional review boards of the US Centers for Disease Control and Prevention and Peking University Health Science Center. Verbal informed consent was obtained from all subjects. Verbal consent was witnessed and formally recorded.

\section{References}

1. Wallingford JB, Niswander LA, Shaw GM et al. (2013) The continuing challenge of understanding, preventing, and treating neural tube defects. Science 339, 1222002.

2. Blencowe H, Cousens S, Modell B et al. (2010) Folic acid to reduce neonatal mortality from neural tube disorders. Int $J$ Epidemiol 39, Suppl. 1, i110-i121.

3. Liu J, Jin L, Li Z et al. (2018) Prevalence and trend of isolated and complicated congenital hydrocephalus and preventive effect of folic acid in northern China, 2005-2015. Metabol Brain Dis 33, 837-842.

4. Jimenez AR, Naz N \& Miyan JA (2019) Altered Folate binding protein expression and Folate delivery are associated with congenital hydrocephalus in the hydrocephalic Texas rat. I Cereb Blood Flow Metab 39, 2061-2073.

5. Kahle KT, Kulkarni AV, Limbrick DD, Jr. et al. (2016) Hydrocephalus in children. Lancet (London, England) 387, 788-799.

6. Jeng S, Gupta N, Wrensch M et al. (2011) Prevalence of congenital hydrocephalus in California, 1991-2000. Pediatr Neurol 45, 67-71.

7. Isaacs AM, Riva-Cambrin J, Yavin D et al. (2018) Age-specific global epidemiology of hydrocephalus: systematic review, meta-analysis and global birth surveillance. PLOS One 13, e0204926.

8. Berry RJ, Li Z, Erickson JD et al. (1999) Prevention of neuraltube defects with folic acid in China. China-U.S. collaborative 
project for neural tube defect prevention. NEngl J Med $\mathbf{3 4 1}$, $1485-1490$

9. Naz N, Jimenez AR, Sanjuan-Vilaplana A et al. (2016) Neonatal hydrocephalus is a result of a block in Folate handling and metabolism involving 10-formyltetrahydrofolate dehydrogenase. $J$ Neurochem 138, 610-623.

10. Reynolds E (2006) Vitamin $\mathrm{B}_{12}$, folic acid, and the nervous system. Lancet Neurol 5, 949-960.

11. Liu J, Li Z, Ye R et al. (2019) Folic acid supplementation and risk for congenital limb reduction defects in China. Int $J$ Epidemiol 48, 2010-2017.

12. Li S, Moore CA, Li Z et al. (2003) A population-based birth defects surveillance system in the People's Republic of China. Paediatr Perinatal Epidemiol 17, 287-293.

13. Garne E, Loane M, Addor MC et al. (2010) Congenital hydrocephalus - prevalence, prenatal diagnosis and outcome of pregnancy in four European regions. Eur J Paediatr Neurol 14, 150-155.

14. Dewan MC, Rattani A, Mekary R et al. (2018) Global hydrocephalus epidemiology and incidence: systematic review and meta-analysis. J Neurosurg 130, 1039-1408.

15. Dai L, Zhou GX, Miao L et al. (2006) Prevalence analysis on congenital hydrocephalus in Chinese perinatal from 1996 to 2004. Zhonghua Yu Fang Yi Xue Za Zhi 40, 180-183.

16. Yi L, Wan C, Deng C et al. (2017) Changes in prevalence and perinatal outcomes of congenital hydrocephalus among
Chinese newborns: a retrospective analysis based on the hospital-based birth defects surveillance system. BMC Pregnancy Childbirth 17, 406.

17. Ren A, Zhang L, Hao L et al. (2007) Comparison of blood Folate levels among pregnant Chinese women in areas with high and low prevalence of neural tube defects. Public Health Nutr 10, 762-768.

18. Liu J, Gao L, Zhang Y et al. (2015) Plasma Folate levels in early to mid pregnancy after a nation-wide folic acid supplementation program in areas with high and low prevalence of neural tube defects in China. Birth Defects Res Part A, Clin Mol Teratol 103, 501-508.

19. Hu S, Wang L, Liu N et al. (2019) Analysis of L1CAM gene mutation in pedigrees with $\mathrm{X}$-linked genetic hydrocephalus. Zhonghua Yi Xue Yi Chuan Xue Za Zbi 36, 465-467.

20. Cains S, Shepherd A, Nabiuni M et al. (2009) Addressing a Folate imbalance in fetal cerebrospinal fluid can decrease the incidence of congenital hydrocephalus. $J$ Neuropathol Exp Neurol 68, 404-416.

21. Stempak JG (1965) Etiology of antenatal hydrocephalus induced by folic acid deficiency in the albino rat. Anat Rec 151, 287-295.

22. Li Z, Ye R, Zhang L et al. (2014) Periconceptional folic acid supplementation and the risk of preterm births in China: a large prospective cohort study. Int $J$ Epidemiol $\mathbf{4 3}$, $1132-1139$. 